$\xi=$ 圈

\title{
Internet of things mathematical approach for detecting brain tumor
}

\author{
Noor Kareem Jumaa ${ }^{1}$, Auday A.H. Mohamad ${ }^{1}$ *, Sameer Hameed Majeed ${ }^{1}$ \\ ${ }^{1}$ Computer Technology Engineering Department, Al-Mansour University College, Baghdad, Iraq \\ *Corresponding author E-mail: auday.mohamad@ieee.org
}

\begin{abstract}
Brain is highly important organ which makes us able to walk, breath, and all other activities; without brain lives can't do all of that. The importance of brain functions made it critical to make any not precisely measured medical action. Currently; computer vision is very important in medical field, where it helps specialists to precisely diagnose and take the right decision before making surgeries. This article worked on accommodating the technology of internet of things (IoT) for serving brain medicine specialist in the field of identifying the need of making surgeries depending on magnetic resonance imaging (MRI) images. Support Vector Machine (SVM) algorithm is used to detect brain tumor and segment it from MRI morphological images. Putting SVM on IoT Thingspeak platform will help brain specialist to diagnose MRI images that are received from MRI computerized system online. The obtained results are compared with same algorithm implemented locally with assist of Matlab program version R2017a.
\end{abstract}

Keywords: Internet of Things; Health care; Brain tumor; MRI; SVM.

\section{Introduction}

Brain is the parts of human body that controlling action of the body parts. Abnormal cells growth of brain leads to brain tumor. Basically, tumor refers to the cells uncontrolled multiplication (i.e. tumors are atypical cells that multiplying out of control.). A cell rapidly divided from a micro calcification, lump, and distortion referred to a tumor. These may differ in size, type, and location [1], [2].

Brain tumor is one of the diseases that seriously cause death among people. A tumor is an unstructured growth of organs in any organ of the human body. It is any mass that results from an abnormal and an unwanted accretion of brain's cells [1,2, and 3].

There are more than 100 different types of brain tumors; but, mainly brain tumors may be classified to the following types: [3], [4]

Benign tumor.

Pre-Malignant tumor.

Malignant tumor

A benign tumor is a tumor that does not extend suddenly; it does not extend to non-adjacent tissues and does not affect its neighboring healthy tissues. Moles are the common example of benign tumors. A premalignant tumor is a pre-carcinogenic phase and it may be classified as a disease, if it is not treated.

Malignant neoplasm is a type of tumor that gets worse over time and at last results is the death of the infected person; tumor cells are presented within the skull and grow within skull which called primary tumor.

Malignant brain tumors are incipient brain tumors. The tumor appears outside the skull and enters the area of the skull called the secondary tumor.

Metastatic tumors are good example of secondary tumor. The tumor takes its place in the skull and interferes with the normal operation of the brain. The brain tumor converts to the skull and increases pressure on the brain. The discovery of the tumor is the first step in treatment [3, 4].

"Detection of the brain tumor is one of the most challenging tasks in the medical image segmentation, since brain images are complicated and tumors can be analyzed only by expert physicians. In brain tumor diagnosis, the important task is to determine the exact location, orientation, and area of the abnormal tissues" [5].

"The important task of brain tumor diagnosis is the exact location, orientation and area of abnormal tissues" [5].

MRI of the brain can be useful in analysis of pathology, presurgical planning, and computer integrated surgery [5, 6].

This article worked on accommodating the technology of internet of things (IoT) for serving healthcare systems.

IoT has no universe definition; different definitions have been used by different references presented by different foundations or research groups to describe the IoT term.

IoT can defined as the communication between things or (physical and logical) objects organized with network to extend to the Internet since the Internet is the communication network between these objects. IoT is a public term of the objects or things that have intelligent interfaces and actively interact [7].

Also in IEEE communication journal, IoT has defined as "a framework wherein all things have a representation and existence in the internet. IoT goals for offering new applications and services bridge both physical and virtual worlds" [8].

For the purpose of this article, IoT defined as: a technology of machine talking to each other which is called "device-to-device" communication or "machine-to-machine" communication (M2M), where M2M communication is described in [8] deeply.

The aim of this article is to propose a software system that deals with brain cancer detection based on support vector machine (SVM) classifier implemented at IoT Thingspeak Platform. It deals with MRI Images of infected brains and detects the tumor in brain. 
SVM is a far improved supervised machine learning algorithm. SVM is a classifier used for classification and regression purposes. The main objective of SVM classifier is to examine set of inputs and classify them accordingly. SVM has two types of margins: Hard margin and Soft margin. Hard margin is the one which separates the two classes without error while the Soft margin is classifying the two classes by allowing error [4, 9]. SVM algorithm can be used in different fields such as face detection, Gait recognition, hand geometry, palm print verification, fingerprint recognition, and iris recognition [9].

The contribution of this paper in the field of IoT is to providing the SVM algorithm as an analytic tool in IoT platform to detect brain tumors.

The article is arranged in 8 sections. Section 2 a review of the related works, Section 3 shows an overview of the most existing brain tumor detection methods, IoT Thingspeak platform is described in section 4 , section 5 presents the proposed tumor detection system, the results have been reviewed in section 6 , section 7 presents the discussion, and the article's conclusions have been presented in section 8 .

\section{Review of related works}

In [4], a hybrid method of support vector machine and fuzzy set classification has been proposed for classification of brains tumor. Results of this paper are examined by an experiment on tumor brain MR images obtained from the internet brain Segmentation repository and diagnostic centers. After applying the SVM classifier on the brain MRI images, important results for classification of brain MRI image have been obtained.

In [6], an IoT system is proposed for health care systems based on RFID technology. This work was supported by the National Natural Science Foundation (NSF) of Peoples Republic of China and the Ministry of Education fund for doctoral disciplines in Higher Education.

A brain tumor detection system is proposed in [10] based on image segmentation and SVM algorithm. The segmentation has been implemented using K-means. The tumor images are divided in Malignant or Benign classes. After identification the image and the feature of it, images are added into the database of the SVM to increase system accuracy.

In [11], IoT based healthcare systems have been survived. This paper includes the webbed health care and that has the complete base of internet of things.

\section{Overview of the most existing methods}

This section demonstrates the most common methods of detection and identification the brain tumor over the MRI images. The following subsections describe briefly the methods: histogram thresholding, region growing, and clustering [4], [12].

\subsection{Histogram thresholding [4], [12]}

Histogram thresholding algorithm (HT) is a simple method used for image processing algorithms; it is used for automatic image thresholding. Like "Iterative selection thresholding method" and "Otsu's Method", this is a histogram based thresholding method. This method proposes that the image is divided into two main categories: background and foreground. The HT tries to obtain the minimum level that divides the histogram into two categories. The histogram threshold is used by the background, extraction of the objects, and image segmentation methods to obtain a range of pixel values that should match to what is required to be selected from the image. A thresholding is a method used to convert a color images or grayscale to a binary images based on threshold values. The histogram shows the frequency of the channel's density values in an image. The minimum and maximum values can be applied using an automatic threshold method or by moving arrows above the histogram.

\subsection{Region growing [4], [12]}

Region growing defined as a region based image segmentation technique. Also; it is categorized as a pixel based image segmentation technique. This method is used to segment examines pixel neighbors of the initial seed points and determine whether the neighboring pixels should be added to the region. Images that have clear edges will have a good segmentation results by using the region growing segmentation method. Only a small number of seed points are needed to represent the property wanted to be extract, and then grow the region. The seed points and the criteria can be determined.

\subsection{Clustering}

Clustering based segmentation techniques are "a set of objects in such a way that objects in the same cluster have higher degree of similarity to each other than to those in other clusters" [4].

Clusters is defined as "contiguous regions of a multidimensional space containing relatively high density of points, separated from other such regions containing relatively low density of points". Image clustering is the process of grouping the pixels rendering to some characteristics like intensity. Clustering lying within two types: hard clustering and soft clustering. In hard clustering, elements of data are belonging to only one group and the membership value of belonging to the cluster is exactly one, while in the soft clustering, the elements of data are belonging to more than one group and membership value belonging to a cluster from 0 to 1 [4], [12].

Clustering methods have two types: K-Mean and Fuzzy clustering; both methods are discussed deeply in [4].

\section{IoT Thingspeak platform [13]}

Thingspeak is an open source IoT cloud platform. It was launched in 2010 by ioBridge as a supporting service of IoT applications. "Thingspeak is an IoT analytics platform service that allows you to aggregate, visualize and analyze live data streams in the cloud". Thingspeak gives an instant visualization of data that aggregated by the devices also, it is used for prototyping and proofing the concept of IoT system that demand analytics.

Thingspeak has many capabilities; some of its key capabilities include the following features:

- Easy configuration: configure devices for sending data to Thingspeak using general IoT protocols.

- Visualization: visualize collected sensor data in real-time.

- Aggregation: aggregate data on request from the sources of third party.

- Analysis: run the automatic IoT analytics based on events or schedules.

- Prototyping: build and prototype IoT systems without servers setting up or web software developing.

- Automation: automatically manipulate the data and communicate by using third party services like Twitter or Twilio. A mash up of various data channels can perform using Thingspeak and better meaningful data can make. Also, Thingspeak permits the user to work on environment by either sending notification or triggering the IP connected thing in environment.

\section{Proposed system}

The proposed system consists of several of stages:

- Collecting brain MRI images.

- Share MRI images to a central secure database website.

- Reading data from the database of website by Thingspeak platform.

There are number of processes are made at this stage using Matlab capabilities at Thingspeak platform. These processes are 
de-noising of the MRI images, skull striping, and segmentation and training the SVM classifier.

By implementing the above said stages the doctor could monitor the patient's case through the cloud. This methodology helps the doctor at the pre surgery step in order to monitor which part of the brain to be moved according to the tumor location.

The block diagram of the suggested system is presented in Figure 1 .Typically; the medical monitoring field necessitates more manual capability to observe patient's health. This is the most forthcoming issues in the medical area. In the case of patient's health is not checked in appropriate time, it leads to destructive situations.

\subsection{Central secure database website}

The main role of central secure database website is to collect MRI images published from the computerized MRI imaging device via internet service provider according to its timestamp (patient ID is high related to data timestamp). This site serves as a broker between the computerized MRI imaging device and the IoT analytic tool (Thingspeak platform).

\subsection{Thingspeak's analytic}

The analytic power of Matlab functions are adopted by Thingspeak IoT platform [13]. Thingspeak Matlab analysis functions import MRI images from the secure website broker. Brain tumor detection was done using SVM algorithm. Accordingly, the doctor can have visual access to the data stream via Thingspeak platform with support of automatic cloud based diagnosing for the patient status by categorizing the MRI images.

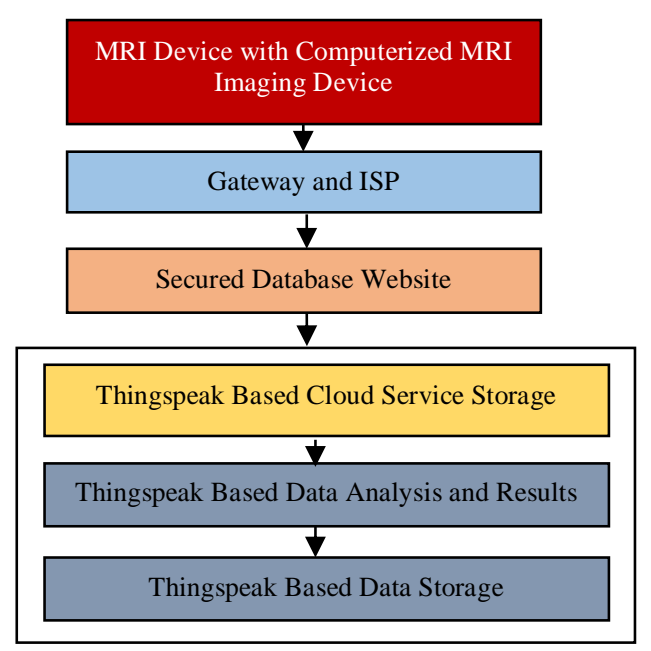

Fig. 1: Block Diagram of the Proposed System.

\section{System implementation and results}

The proposed IoT based detecting brain tumor with Thingspeak platform is composed of three main parts as in Figure.1. The first part is computerized MRI imaging device. The MRI images are collected and saved as JPG file and forwarded to a central website broker (second part). For experimental study, a website with domain name audaymohamad.com from TMDHosting has been created. Three MRI brain images shown in Figure 2 are forwarded and saved in audaymohamad.com website.

The third part of the suggested system is Thingspeak platform; in this part the analytic power of Matlab program is used to implement the following:

\subsection{Images attainment}

Brain MRI images were aggregated from the broker website audaymohamad.com. Those images with size of $256 \times 256$ pixels. Figure 3 shows the three original test images shown in Figure 2 at Thingspeak data base and they are ready for classification process.

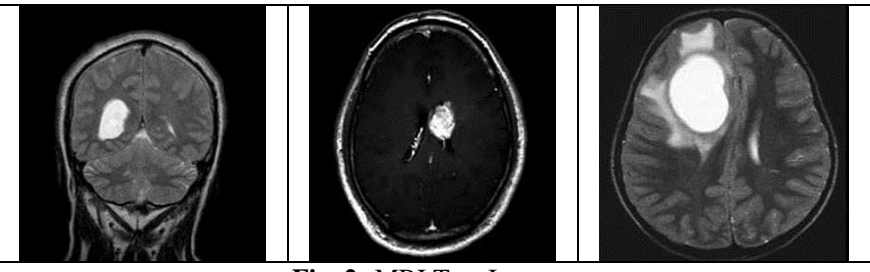

Fig. 2: MRI Test Images.

MATLAB Plot Output
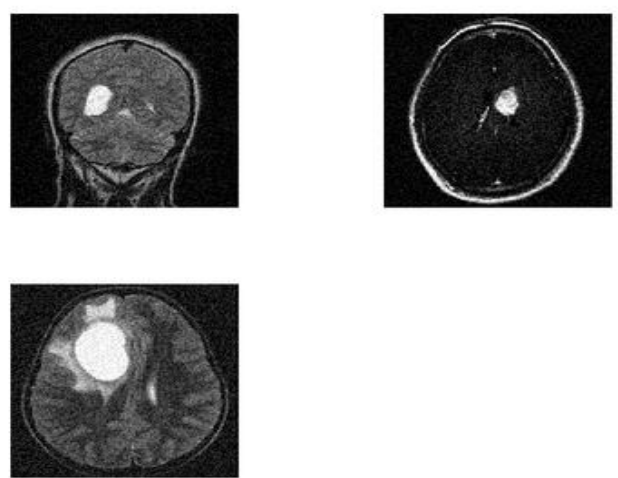

Fig. 3: MRI Test Images at Thingspeak Platform.

\subsection{De-noising MRI images}

Using De-noising technique increases and improves the qualities of MRI images. It is an important to enhance the MRI images for viewers, so; precise results are achieved. The MRI images shown in Figure 2 have been filtered using the discrete PDE (Partial Differential Equation) with the parameters:

Number of iteration $=10$.

delta_t $=1 / 7$.

kappa $=15$.

Option $=2$.

Where Number of iteration is performing how many the MRI image has been filtered from noise.

delta_t is the discrete PDE solution factor.

Kappa is the Diffusion Equation factor.

Option is dedicate the type of the Diffusion Equation.

Figure 4 shows the removing noise of test MRI images implemented in local computer with locally installed Matlab program version R2017a.

Figure 5 shows the removing noise of test MRI images implemented in cloud based on Thingspeak platform.

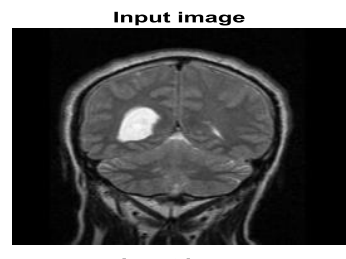

Input image
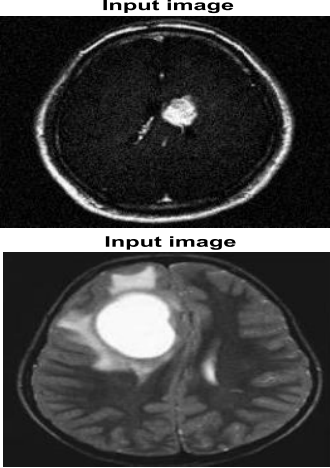

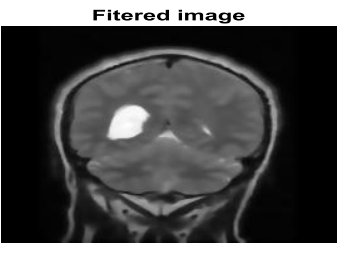

Fitered image
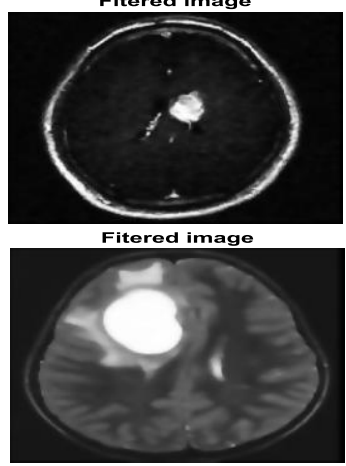

Fig. 4: Original and Filtered Images Implemented Locally. 
MATLAB Plot Output
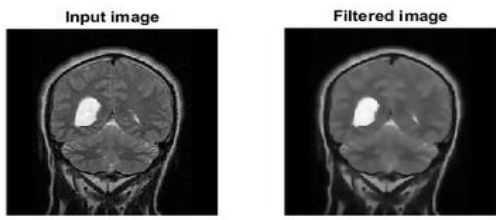

MATLAB Plot Output
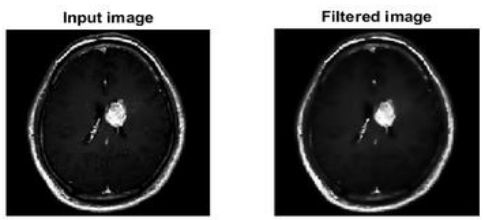

MATLAB Plot Output
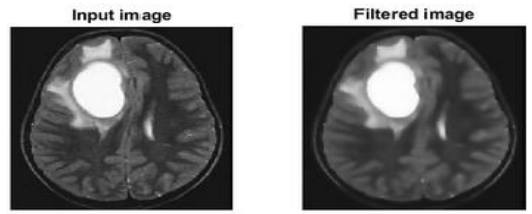

Fig. 5: Original and Filtered Images at Thingspeak Platform.

Figure 6 shows the input filtered image, initial mask image and the segmented image implemented in local computer with locally installed Matlab program version R2017a.

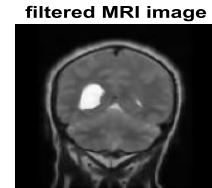

filtered MRI image

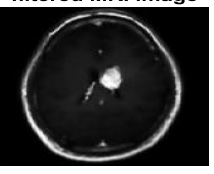

filtered MRI image

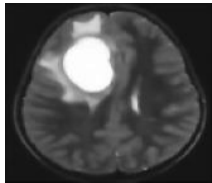

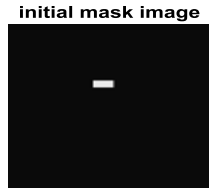

initial mask image

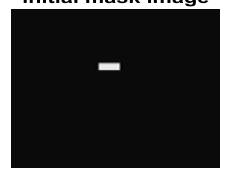

initial mask image

-

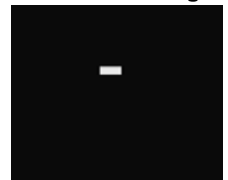

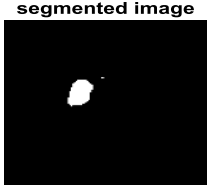

segmented image

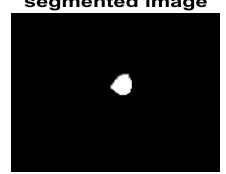

segmented image

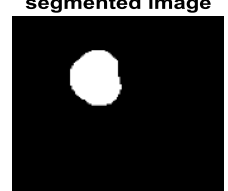

Fig. 6: Input Filtered, Initial Mask and the Segmented Images Implemented Locally.

\subsection{Support vector machine (SVM)}

It is a supervised learning technique. SVM is a good technique for classification and analysis of data. Even in large data, classifier based on SVM has a good learning speed. SVM is based on the concept of decision-making levels where the level of resolution separates a group of elements that have different membership in the class.

The SVM that in this article is used as a segmentation method and has the parameters of Alpha $=0.2$, grayscale filtered RM image and binary initial mask image for the filtered image in Figures 4 and 5. The input image is with $128 \times 128$ size and the initial mask image is with the same size.

Figure 7 shows the input image, initial mask image and the segmented image implemented in cloud based on Thingspeak platform

\section{MATLAB Plot Output}
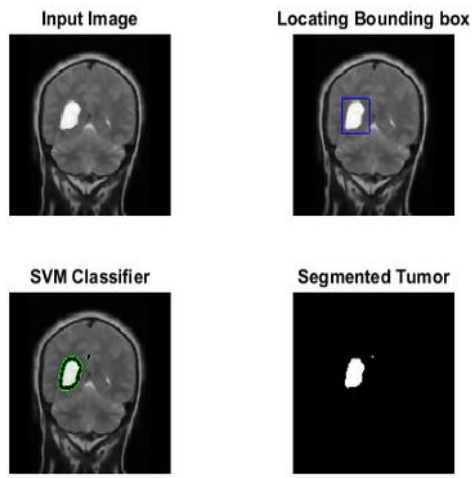

MATLAB Plot Output
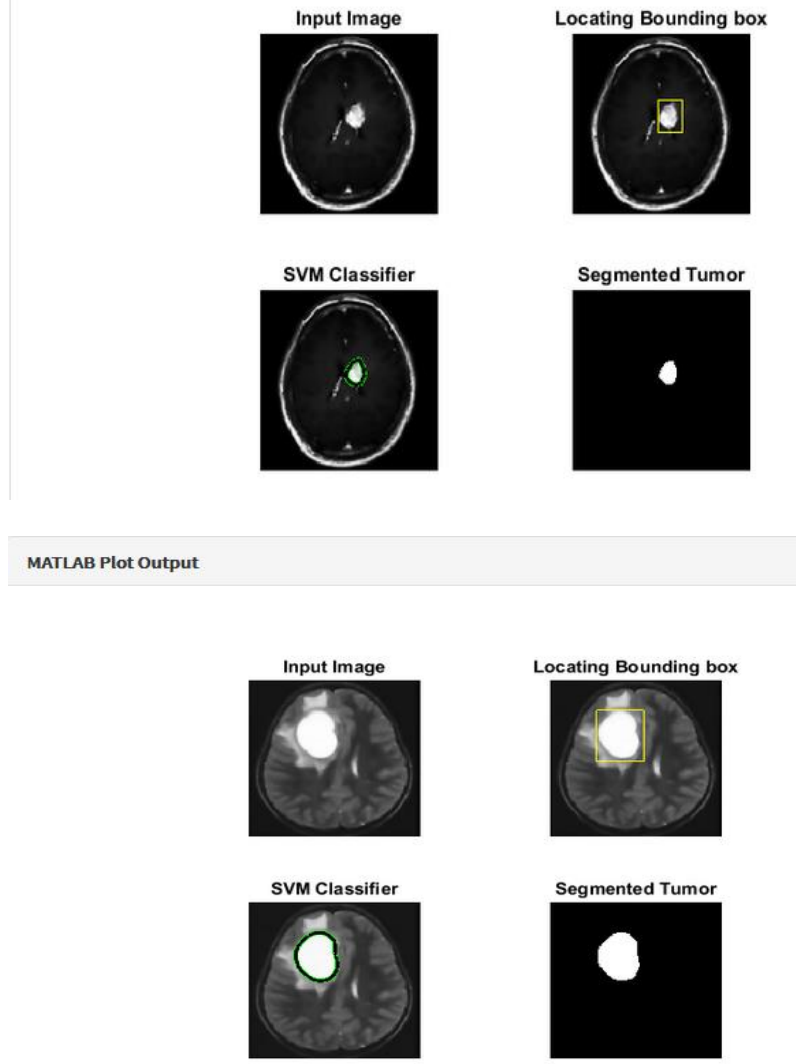

Segmented Tumor

Fig. 7: Input, Initial Mask and Segmented Images Implemented at Thingspeak Platform. 


\section{Discussion}

Based on above methods and results, MRI images are published to the Thingspeak's platform to test the work process of the proposed system. The SVM is used as a classifier algorithm with a set of preprocessing operations. Those algorithms are implemented at cloud level depends on cloud Matlab functions built inside Thingspeak platform. A sample of MRI images for infected brains (shown in Figure 2) are published successfully to Thingspeak platform as shown in Figure 3. The SVM algorithm for tumor detection has been implemented locally with Matlab program R2017a and at Thingspeak platform as shown in Figures 6-7 respectively. So, based on above results, we conclude that the proposed online classifier algorithm has excellent processing results as compared with the same algorithm implemented locally.

Three MRI images of infected brains are tested using the proposed system of this paper and all of these images have been classified as brains with tumor and the efficiency of the proposed system was $100 \%$.

\section{Conclusions}

Internet of Thing (IoT) has been implemented as a smart objects and stuff that communicate with each other through the Internet. Applied the IoT as smart applications that communicate with users via the cloud is still an area for researches. In this paper, IoT has been applied as a technology for a healthcare system. Also, IoT technology was used to run the SVM classifier as an artificial intelligent over the cloud. Thus, this paper presents one of the modern IoT based artificial intelligent through an IoT platform (exactly Thingspeak IoT platform). And as mentioned before, the efficiency of the proposed system is $100 \%$.

\section{References}

[1] Neha Rani and Sharda Vashisth,"Brain Tumor Detection and Classification with Feed Forward Back-Prop Neural Network", International journal of computer applications, Vol. 146, No.12, (2016).

[2] Tanvi Gupta, Pranay Manocha, Tapan K. Gandhi, R.K. Gupta, and B.K. Panigrahia (2002), Tumor classification and segmentation of MRI brain images, Fortis memorial research institute, Gurgaon, India.

[3] Miss. Priyanka Katti and Mr. V. R. Marathe, "Implementation of Classification System for Brain Tumor using Probabilistic Neural Network", International journal of advanced research in computer and communication engineering, Vol. 4, Issue. 10, (2015).

[4] Dipali B.Birnale and S. N. Patil,"Brain Tumor MRI Image Segmentation using FCM and SVM Techniques", International journal of engineering science and computing, Vol. 6, No. 12, (2016).

[5] Jia Li, Dhiraj Joshi, and James Z. Wang, Stochastic modeling of volume images with a 3-D hidden markov model, US National Science Foundation, USA

[6] Sajjad Hussain Talpur,"The Appliance Pervasive of Internet of Things in Healthcare Systems", International journal of computer science issues, Vol. 10, Issue. 1, No. 1, (2013).

[7] Hyo-Sik Ham, Hwan-Hee Kim, Myung-Sup Kim, and Mi-Jung Choi, "Linear SVM-Based Android Malware Detection for Reliable IoT Services", Journal of applied mathematics, Vol., (2014).

[8] Internet Society (2015), the internet of things: an overview, available online: www.internetsociety.org.

[9] Nandhini.K and B.Sanithi,"Retrospection of SVM classifier", Jour nal of theoretical and applied information technology, Vol. 38, No. 1, (2012).

[10] Swapnil R. Telrandhe, Amit Pimpalkar, and Ankita Kendhe, "Implementation of Brain Tumor Detection using Segmentation Algorithm \& SVM", International journal on computer science and engineering, Vol. 8, No.7, (2016).

[11] Ms. Shinde Sayali P. and Ms. Phalle Vaibhavi N,"A Survey Paper on Internet of Things based Healthcare System", International advanced research journal in science, engineering and technology, Vol. 4, Special Issue 4, (2017).

[12] Richa Aggarwal and Amanpreet Kaur,'Efficient Analysis of Brain Tumor Detection and Identification Using Different Algorithms",
International journal of sciences \& research technology, Vol. 3, (2014).

[13] www.thingspeak.com. 